\title{
LOS IMPACTOS ECONÓMICOS, SOCIALES $Y$ MEDIOAMBIENTALES NEGATIVOS EN EL ECOTURISMO: UNA REVISIÓN DE LA LITERATURA
}

\author{
Francisco Orgaz Agüera ${ }^{1}$ \\ Universidad Tecnológica de Santiago (UTESA), República Dominicana
}

http://dx.doi.org/10.5209/rev_NOMA.2014.v42.n2.48781

\begin{abstract}
Resumen.- El ecoturismo se configura como una tipología turística que se desarrolla en contacto con la naturaleza. Este turismo ayuda a mejorar el desarrollo socioeconómico de las comunidades locales, a la vez, que fomenta la conservación de los recursos naturales y el respeto hacia el medio ambiente. Aunque, esta tipología de turismo también genera importantes impactos negativos. El objetivo de esta investigación es analizar los diferentes impactos económicos, sociales y medioambientales que puede provocar el desarrollo de la actividad ecoturística. La metodología de esta investigación ha consistido en una revisión de la literatura científica. Los resultados muestran que la actividad ecoturística puede generar diversos impactos negativos en el destino.
\end{abstract}

Palabras clave.- Ecoturismo, Desarrollo Económico, Desarrollo Sostenible, Recursos Naturales, Medio Ambiente

\begin{abstract}
Ecotourism is configured as a type of tourism that it is developing in contact with the nature. This tourism also helps to improve the socio-economic development of local communities and promotes the conservation of natural resources and respect for the environment. Although, ecotourism also generates significant negative impacts. The objective of this research is to analyze different economic, social and environmental impacts that ecotourism can cause on the destination. The methodology has consisted of a review of the scientific literature. The results show that ecotourism can generate various negative impacts on the destination.
\end{abstract}

Keywords: Ecotourism, Economic Development, Sustainable Development, Natural Resources, Environment

\footnotetext{
${ }^{1}$ Profesor en Turismo, Administración de Empresas y Medio Ambiente en la Universidad Tecnológica de Santiago (UTESA), República Dominicana. Doctorando en Turismo por la Universidad de Sevilla (España). Máster en Dirección y Planificación del Turismo por la Universidad de Sevilla. Diplomado en Turismo por la Universidad de Córdoba (España). Sus líneas de investigación son el turismo, medio ambiente y el desarrollo sostenible. Email: franorgaz@hotmail.com
} 


\section{Introducción.}

El turismo es uno de los principales sectores económicos a nivel mundial. En este aspecto, la demanda turística está buscando nuevas experiencias lejos de los lugares masificados, donde puedan encontrar rasgos éticos, naturales y reales a través de la autenticidad de un destino (Yeoman et al., 2007). Además, las mejoras que se están produciendo desde hace años en las comunicaciones, tanto a nivel de infraestructura como de tecnología, están aumentando las oportunidades de la demanda turística para realizar viajes (Castillejo Canalejo et al., 2011), prefiriendo sobre todo realizar viajes más cortos en el tiempo pero con una mayor frecuencia durante el año (LópezGuzmán et al., 2013), con el objetivo de dar respuesta a los diferentes gustos y necesidades que tienen actualmente los turistas.

Esto está provocando que estén apareciendo nuevas tipologías turísticas a través de elementos relacionados con la cultura (Molina Collado et al. 2007; Orgaz Agüera, 2013), naturaleza (Budowskl, 1976; Moral Cuadra y Orgaz Agüera, 2012), gastronomía (Navarro y Schlüter, 2010; López-Guzmán y Sánchez Cañizares, 2012), industria (Castillejo Canalejo et al., 2011; Contreras et al., 2013), religión (Poncelaii, 2010; Millán Vázquez de la Torre et al., 2012), etc., que están generando nuevas vías de ingresos para las comunidades locales, a través de la creación de empleo y la puesta en marcha de nuevas empresas en el destino. Por tanto, según Castillejo Canalejo et al., (2011), la aparición de estas tipologías turísticas están provocando que los criterios para clasificar estos tipos de turismo sean cada vez más variados.

En este sentido, algunos autores clasifican el turismo en dos grupos (Vargas Sanchez 2007, Castillo Canalejo et al. 2011): Turismo de masas y turismo alternativo. Este segundo grupo se caracteriza por desarrollar un turismo de forma más sostenible, donde se tienen en cuenta aspectos como el desarrollo socioeconómico de las comunidades locales, las potencialidades de los recursos naturales, culturales y patrimoniales locales, y la conservación y respeto hacia los recursos naturales y el medio ambiente.

Así, dentro de este último grupo se clasifica el ecoturismo. Esta tipología turística busca a través de la explotación de los recursos naturales y culturales, desarrollar las comunidades locales y conservar los recursos naturales y culturales del destino.

Pero, esta forma de turismo puede generar diversos impactos en el destino ecoturístico, lo que puede provocar diferentes problemas en el lugar. En este sentido, el objetivo de esta investigación es analizar los principales impactos negativos de índole económica, social y medioambiental que puede generar el desarrollo de la actividad ecoturística en un destino geográfico.

La metodología empleada, para la realización de esta investigación, ha consistido en una revisión de la literatura científica sobre los estudios de ecoturismo en diversos destinos internacionales. Esta metodología ha sido 
utilizada por otros investigadores en sus estudios (López-Guzmán et al., 2013), dando así respuesta a la necesidad que existen en los diferentes campos científicos de realizar una periódica compilación de la literatura existente sobre el tema (Getz y Brown, 2006).

Para cumplir con los objetivos marcados en este trabajo, este artículo se ha estructurado tras esta introducción, en un segundo apartado donde se hace una introducción sobre la actividad ecoturística. Posteriormente, en un tercer apartado, se desarrollan los impactos del ecoturismo a nivel económico, social y medioambiental. En un cuarto apartado se realizan las conclusiones de esta investigación. Finalmente, en un quinto apartado, se muestran las referencias bibliográficas consultadas para la elaboración de este artículo.

\section{Introducción al ecoturismo.}

El concepto de ecoturismo viene siendo objeto de definición desde hace pocos años (Sandoval 2007). Si bien, han sido muchos los autores e investigadores que han definido el término ecoturismo. Así, Fennell (2001) encontró 85 conceptos diferentes de ecoturismo en la literatura científica.

En este sentido, la definición que ha tenido más aceptación entre la comunidad científica ha sido la elaborada por Ceballos-Lascurain (1992), el cuál definía ecoturismo como aquella modalidad turística ambientalmente responsable consistente en viajar o visitar áreas naturales relativamente sin disturbar, con el fin de disfrutar, apreciar y estudiar los atractivos naturales (paisaje, flora y fauna silvestres) de dichas áreas, así como cualquier manifestación cultural (del presente y del pasado) que puedan encontrarse ahí, a través de un proceso que promueve la conservación, tiene bajo impacto ambiental y cultural, y propicia un involucramiento activo y socioeconómicamente benéfico de las poblaciones locales.

Esta definición fue aceptada posteriormente por diferentes organismos e instituciones internacionales. En este aspecto, la Organización Mundial del Turismo (OMT) elaboró una serie de características aplicables a esta forma de turismo (OMT 2002):

a) El ecoturismo se desarrolla en contacto con la naturaleza, y la motivación principal del ecoturista es la observación de la misma y el disfrute de la cultura dominante en las áreas naturales.

b) El ecoturismo se compone por elementos de interpretación y de educación.

c) El viaje suele estar organizado por pequeñas empresas especializadas, pequeñas y de propiedad local, y se dirigen a grupos pequeños.

d) El desarrollo de la actividad turística busca no producir impactos negativos en el medio natural ni en la sociedad y cultura dominante en las áreas naturales.

e) La actividad ecoturística mejora la protección de las áreas protegidas: Genera recursos económicos para la población local, las empresas 
locales y demás stakeholders ${ }^{2}$ locales; ofrece oportunidades de empleo a la población local; y, crea concienciación y sensibilización de la población local y los turistas hacía los recursos naturales y culturales.

Así, la actividad ecoturística ha sido objeto de investigación por numerosos autores y académicos (Stronza y Gordillo 2008, Jing y Fucai 2011, Lu y Stepchenkova 2012, Castellanos Verdugo y Orgaz Agüera, 2013). En este sentido, para diversos autores, el ecoturismo está compuesto por diferentes componentes, que sin los cuales no podría desarrollarse:

a) La demanda ecoturística, que son aquellos turistas que desarrollan actividades en contacto con la naturaleza (Ingram y Durst, 1989; Fennell y Eagles, 1990; Eagles, 1992).

b) Las actividades ecoturísticas, como son las observación de aves o birdwatching, la observación de ballenas y tortugas marinas, recorridos por la selva, safaris fotográficos, buceo y snorkeling, entre otras (Pérez de las Heras, 2003).

c) Los destinos ecoturísticos o aquellas áreas naturales protegidas que tienen gran belleza natural e interés ecológico (Puertas Cañaveral, 2007).

d) La infraestructura ecoturística que suele estar formada por las áreas recreativas, aulas de naturaleza, carril de ciclo-turismo, centro de visitantes, miradores, senderos y observatorios, entre otros (Puertas Cañaveral, 2007).

e) Los guías de ecoturismo o personas encargadas de explicar los aspectos naturales y culturales del área natural a los ecoturistas (Fennell y Eagle, 1990).

f) Los stakeholders del ecoturismo o todos aquellos actores que pueden afectar y ser afectados por las actividades del ecoturismo (Ziffer, 1989; Valentine, 1993).

Por lo tanto, el ecoturismo se configura como una actividad turística en contacto con la naturaleza, que puede generar impactos positivos en el destino, pero que también, puede provocar diversos impactos negativos en el área ecoturística.

\section{Impactos negativos del ecoturismo.}

Los impactos en el ecoturismo son generados durante el desarrollo de la actividad ecoturística, y pueden ser de índole económica, social o medioambiental. A su vez, estos impactos pueden clasificarse en positivos (beneficios) o negativos (costos). A continuación se analizan los diversos

\footnotetext{
${ }^{2}$ En este contexto, los stakeholders hacen referencia a aquellos agentes que pueden afectar o ser afectados por el desarrollo de la actividad ecoturística. Este término fue utilizado por primera vez por Freeman (1984), posteriormente, muchos han sido los autores que lo han llevado al campo del turismo (Álvarez et al., 2001; Cespedes et al., 2003; Sheehan et al., 2007).
} 
impactos negativos o costos de índole económica, social y medioambiental que pueden ser provocados por el desarrollo de la actividad ecoturística.

\subsection{Impactos económicos.}

Según Sancho (1998), los impactos económicos negativos de la actividad turística son aquellos derivados de los costes provocados por las fluctuaciones de la demanda turística, la inflación derivada de la actividad turística y las distorsiones en la economía local. Todos estos son impactos del turismo en general, y como tal, son aplicables también de forma particular al ecoturismo.

Además, Puertas Cañaveral (2007) cita dos más que deben ser considerados dentro de la actividad ecoturística. En primer lugar, el hecho de que los beneficios financieros reportados por esta tipología turística no reviertan sobre la zona y la comunidad local. Para evitar esto, Puertas Cañaveral (2007) habla de implementar una buena gestión que tenga como finalidad básica la conservación de la sostenibilidad ambiental. En segundo lugar, en el turismo ecológico son fuertes las fluctuaciones económicas, debido a varias razones:

- La estacionalidad de ciertas actividades turísticas en contacto con la naturaleza, que sólo pueden realizarse en determinadas épocas del año.

- La seguridad y estabilidad política de un destino producido por atentados terroristas, hurtos a turistas o virus y/o enfermedades. Esta afecta, sobre todo, a los países en vías de desarrollo.

\subsection{Impactos sociales.}

Para algunos investigadores (Wearing y Neil 2000, Pérez de las Heras 2002, Puertas Cañaveral 2007) existen diversos problemas provocados por las visitas de los turistas a un destino. Estos son aplicables al turismo ecológico en particular, y son los siguientes:

- Aparición de diferencias socioculturales entre la población residente y los turistas.

- Escasa posibilidad de intervención en la toma de decisiones de la puesta en marcha del ecoturismo por parte de las comunidades locales.

- Falta de reconocimiento en la labor desarrollada por parte de la población residente.

- Necesidad de instrumentos que permitan una adecuada evaluación de los diversos impactos ambientales y socio-culturales que se puedan generar en el destino.

- Peligro de establecer la actividad turística como el único sector económico en el área geográfica, provocando impactos negativos en otros sectores económicos del destino.

- Aceleración de los cambios sociales de la población residente en el destino ecoturístico.

- Falta de obtención de beneficios económicos y sociales de los proyectos desarrollados en el área geográfica. 
- Impactos que afectan a la cohesión poblacional, es decir, mientras un grupo apoya el proyecto ecoturístico, otro no está a favor. Esto puede generar diversas manifestaciones sociales en el destino, provocando enfrentamientos entre la población local.

\subsection{Impactos medioambientales.}

El turismo ecológico como actividad turística que se desarrolla en zonas naturales, puede generar diversos daños en el medio ambiente, debido a la fragilidad de los ecosistemas.

En este sentido, según diversos autores (Del Reguero Oxinalde 1994, De las Heras 2002), los impactos negativos hacia el medio natural producidos por el turismo son muy diversos. Estos pueden ser aplicables al caso del ecoturismo y se clasifican en tres apartados (Puertas Cañaveral, 2007):

- Aquellos provocados por la construcción de infraestructuras, equipamientos y servicios: La creación de los servicios mínimo y de alojamiento provoca, en el menor de los casos, un deterioro en el medio natural, que posteriormente se maximiza con el producido por la energía, la utilización y contaminación de agua, y la eliminación de residuos sólidos.

- Aquellos causados por las actividades que realizan los propios ecoturistas: Los ecoturistas, en el menor de los casos y aunque realicen actividades en grupos pequeños, provocan en el medio natural unos impactos negativos, que varían de intensidad según el tipo de actividad que desarrollen (senderismo, safaris, paseos en bicicleta, etc.).

- Aquellos provocados por el número de visitantes que visitan un determinado lugar al mismo tiempo: Mientras mayor sea el grupo que realiza la actividad ecoturística mayor será el impacto que estos visitantes provoquen en el medio natural.

Siguiendo a Puertas Cañaveral (2007), el ecoturismo además puede ocasionar diversos conflictos en los espacios naturales, destacando los siguientes:

- Contaminación ambiental, visual y acústica: La construcción de equipamientos que no estén integrados con el entorno natural y el paisaje, así como la contaminación acústica, pueden llegar a provocar la pérdida y la huida de numerosos animales en la zona. Además, la emisión de gases provocados por los medios de transportes son muy perjudiciales, debido a que emiten sustancias tóxicas y perjudiciales para todos los seres vivos que habitan y visitan la zona.

- Modificación de la flora y fauna: La visita de los ecoturistas a un área natural provoca modificaciones en el comportamiento de la flora y de la fauna (desgaste del suelo, huida de especies de fauna, etc.).

- Explotación de agua y energía: La actividad turística en general es una consumidora potencial de agua y como tal se debe de controlar el consumo de la misma. El consumo de energía provoca una emisión de gases en el área que conlleva a que la calidad ambiental disminuya. 


\section{Conclusiones.}

El ecoturismo se configura como una actividad turística que se desarrolla en contacto con la naturaleza, y que tiene como finalidad mejorar el desarrollo socioeconómico y la conservación de los recursos naturales y patrimoniales, a través de la explotación de los recursos locales.

En este sentido, para que pueda desarrollarse la actividad ecoturística adecuadamente deben existir una serie de componentes: Demanda ecoturística, actividades ecoturísticas, stakeholders del ecoturismo, destinos ecoturísticos, infraestructura ecoturísticas y guías del ecoturismo. Todos estos componentes son los responsables de que se desarrolle de forma adecuada la actividad ecoturística en el destino. En este mismo sentido, la falta de uno de ellos genera que no se desarrolle adecuadamente la actividad ecoturística en el destino.

Como tal, el desarrollo de esta actividad provoca a su vez unos impactos de índole social, económico y medioambiental en el destino ecoturístico y que puede favorecer a la zona o área y a la población local, pero también perjudicar el destino si no se toman medidas concretas que alivien los impactos negativos que provoca la actividad.

En este sentido, cuando la actividad ecoturística no se desarrolla teniendo en cuenta la participación de las comunidades locales, pueden generarse costes negativos en el destino, debido a que este grupo de población no recibe los beneficios económicos por el desarrollo de la actividad ecoturística. Por tanto, el ecoturismo debe desarrollarse bajo la participación en la gestión y planificación de la población local.

A su vez, los impactos económicos negativos pueden generar problemas en la población local, creando conflictos entre los propios habitantes de las comunidades locales o entre la población local y los turistas.

Así, el ecoturismo también puede generar numerosos impactos negativos en el destino, tales como pérdidas o alteraciones de flora y fauna o contaminación, que puede afectar a las poblaciones futuras que habiten o visiten el destino.

Para ello, el ecoturismo debe planificarse de forma adecuada, teniendo en cuenta a las comunidades locales como el eje principal de la actividad ecoturística en el destino, y aplicando conceptos como capacidad de carga y desarrollo sostenible.

A través del desarrollo sostenible se pretende mejorar el desarrollo socioeconómico de las comunidades locales y fomentar la conservación y el respeto hacia la naturaleza y el medio ambiente. Por su parte, con la capacidad de carga se evita que haya más personas de las debidas al mismo tiempo en el recurso o destino ecoturístico. También se hace necesaria la formación en turismo de la comunidad local, con la finalidad de poder desarrollar conceptos y 
habilidades en la población local, con la misión de que puedan trabajar en el sector turístico local.

Por lo tanto, y como conclusión final, a través de la aplicación del concepto de desarrollo sostenible y de capacidad de carga, además de planificar el ecoturismo teniendo en cuenta como eje principal a la población local del destino, se puede contribuir a reducir los numerosos impactos negativos que se producen o se pueden producir con el desarrollo del ecoturismo.

\section{Referencias bibliográficas.}

ÁLVAREZ, M. J.; DE BURGOS, J. y CÉSPEDES, J. J. (2001) “ Grupos de Interés y Estrategias Medioambientales de los Hoteles Españoles". ICE: Tribuna de Economía, no 790, pp. 163-181.

BUDOWSKI, G. (1976) "Tourism and environmental conservation: Conflict, coexistence or simbiosis?", Environmental Conservation, vol. 31, n $\mathrm{n}^{\mathrm{1}}$, pp. $27-$ 31.

CASTELLANOS VERDUGO, M. y ORGAZ AGÜERA, F. (2013) "Potencialidades ecoturísticas de la República Dominicana". TURyDES, Revista de Investigación en Turismo y Desarrollo Local, vol. 6, n 14, pp. 1-15.

CASTILLO CANALEJO, A. M.; LÓPEZ-GUZMÁN, T. y MILLÁN VÁZQUEZ DE LA TORRE, G. (2011) "Delimitación conceptual y consideraciones en torno al turismo industrial minero. TURyDES, Revista de Investigación en Turismo y Desarrollo Local, Vol. 4, No 9, pp. 1-15.

CEBALLOS-LASCURAIN, H. (1992) "Tourism, ecotourism and protected areas: national parks and protected areas". IV Congreso sobre Parques Nacionales y Zonas Protegidas, Caracas, Venezuela, pp. 84-89.

CÉSPEDES, J.; DE BURGOS, J. y ÁLVAREZ, J. M. (2003) "Stakeholders' environmental influence: An empirical analysis in the Spanish hotel industry". Scandinavian Journal of Management, vol. 19, pp. 333- 358.

CONTRERAS, T. J. C., HERNÁNDEZ, I. Z., y SILVA, F. A. B. (2013) "Turismo industrial en la Frontera Norte. Una expectativa de desarrollo para Ciudad Juárez, Chihuahua". Teoría y Praxis, vol. 13, pp. 34-58.

DEL REGUERO OXINALDE, M. (1994) Ecoturismo. Nuevas formas de turismo en el espacio rural. España: Bosch.

EAGLES, P. F. J. (1992) "The travel motivations of Canadian ecotourist". Journal of Travel Research, vol. 2, n 31, pp. 3-7.

FENNELL, D. (2001) "A content analysis of ecotourism definitions". Current Issues in Tourism, vol. 4, pp. 403-421.

FENNELL, D. y EAGLES, P. (1990) "Ecotourism in Costa Rica: A conceptual framework". Journal Park and Recreation Administration, vol. 1, $\mathrm{n}^{\circ} 1$, pp. 23-34. FREEMAN, R. E. (1984) Strategic Management: A stakeholder approach. Boston: Pitman.

GETZ, D. y BROWN, G. (2006) "Benchmarking wine tourism development". International Journal of Wine Marketing, $\mathrm{n}^{\circ}$ 18, vol. 2, pp. 78-97.

JING, Y. y FUCAI, H. (2011) "Research on Management of Ecotourism Based on Economic Models". Energy Procedia, vol. 5, pp. 1563-1567.

INGRAM, C. D. y DURST, P. B. (1989) "Nature oriented tour operators: Travel to developing countries". Journal Travel Research, vol. 2, n² 28, pp. 11-15. 
LÓPEZ-GUZMÁN, T.; RODRÍGUEZ GARCÍA, J. y VIERA RODRÍGUEZ, A. (2013) "Revisión de la literatura científica sobre enoturismo en España". Cuadernos de Turismo, vol. 32, pp. 171-188.

LÓPEZ-GUZMÁN, T. Y SÁNCHEZ CAÑIZARES, S. M. (2012) "La gastronomía como motivación para viajar. Un estudio sobre el turismo culinario en Córdoba". PASOS, revista de turismo y patrimonio cultural, vol. 10, n 5, pp. 575-584.

LU, W. y STEPCHENKOVA, S. (2012) "Ecotourism experiences reported online: Classification of satisfaction attributes". Tourism Management, vol. 33, no 3, pp. 702-712.

MILLÁN VÁZQUEZ DE LA TORRE, M. G.; PÉREZ NARANJO, L. y MARTÍNEZ CÁRDENAS, R. (2012) "Etapas del ciclo de vida en el desarrollo del turismo religioso: Una comparación de estudios de caso". Cuadernos de Turismo, vol. 30, pp. 241-266.

MOLINA COLLADO, A.; MARTÍN-CONSUEGRA NAVARRO, D.; ESTEBAN TALAYA, A. y DÍAZ SANCHEZ, E. (2007) "Segmentación de la demanda turística: Un análisis aplicado a un destino de turismo cultural". Revista de Análisis Turísticom, vol. 4 (2० Semestre), pp. 36-48.

MORAL CUADRA, S. y ORGAZ AGÜERA, F. (2012) "El Turismo Ornitológico: Concepto, Evolución, Características y Mercados Meta. El Caso de Andalucía". $V$ Jornadas de Investigación en Turismo. Universidad de Sevilla, Sevilla, pp. 701-718.

NAVARRO, F., y SCHLÜTER, R. (2010) "El turismo en los pueblos rurales de argentina:¿ Es la gastronomía una opción de desarrollo?". Estudios y perspectivas en turismo, vol. 19, n 6 , pp. 909-929.

OMT. (2002) El mercado español del ecoturismo. Madrid: Organización Mundial del Turismo.

ORGAZ AGÜERA, F. (2013) "Patrimonio y cultura en el Caribe: Presentación y propuestas de promoción y comercialización turística de los museos en Santo Domingo". ASRI: Arte y Sociedad, Revista de Investigación, n 5, pp. 1-10.

PÉREZ DE LAS HERAS, M. (1999) La guía del ecoturismo o cómo conservar la naturaleza a través del turismo. Madrid: Mundi Prensa.

PONCELAII, A. M. F. (2010) "El Santo Niño de Atocha: patrimonio y turismo religioso". Pasos. Revista de turismo y patrimonio cultural, vol. 8, nº 2.

PUERTAS CAÑAVERAL, I. (2007) Ecoturismo en las Reservas de la Biosfera. Granada: Universidad de Granada.

SANCHO, A. (1998) Introducción al turismo. Madrid: Organización Mundial del Turismo.

SANDOVAL, E. (2007) Ecoturismo: Operación Técnica y Gestión Ambiental. México: Trillas.

SHEEHAN, L.; RITCHIE, J. R. B. y HUDSON, S. (2007) "The destination promotion triad: Understanding asymmetric stakeholder interdependencies among the city, hotels, and DMO". Journal of Travel Research, vol. 46, $\mathrm{n}^{\circ} 1$, pp. 64-74.

STRONZA, A. y GORDILLO, J. (2008) "Opiniones comunitarias sobre ecoturismo". Annals of Tourism Research en español, vol. 10, n 1, pp. 26-49.

VALENTINE, P. S. (1993) "Ecotourism and nature conservation: A definition with some recent developments". Tourism Management, vol. 14, n 2, pp. 107115. 
VARGAS SÁNCHEZ, A. (2007) Turismo industrial en la provincia de Huelva: Presente y Futuro. Huelva: Cátedra Cepsa.

WEARING, S. y NEIL, J. (2000) Ecoturismo. Impacto, tendencias y posibilidades. Madrid: Síntesis.

YEOMAN, I.; BRASS, D. y MCMAHON-BEATTIE, U. (2007) "Current issue in tourism: the authentic tourist". Tourism Management, vol. 28, pp. 1128-1138.

ZIFFER, K. (1989) Ecotourism: The uneasy alliance. Washington DC: Ernst and Young, Conservation International. 